\title{
Changes of the Airway Space and the Position of Hyoid Bone after Mandibular Set Back Surgery Using Bilateral Sagittal Split Ramus Osteotomy Technique
}

\author{
Sung-Keun Choi, Ji-Eun Yoon, Jung-Won Cho, Jin-Woo Kim, Sun-Jong Kim, Myung-Rae Kim
}

Department of Oral and Maxillofacial Surgery, Ewha Womans University Medical Center

\begin{abstract}
Purpose: Although there have been several studies of reduced airway space after mandibular setback surgery using the sagittal split ramus osteotomy technique, research on the risk factors for changes of the airway space is lacking. Therefore, this study was performed to examine airway changes and the position of the hyoid bone after orthognathic surgery, and to assess possible risk factors.

Methods: In this retrospective study, 50 patients who underwent posterior displacement of the mandible by the bilateral sagittal split ramus osteotomy technique were included. Changes of the position of the hyoid bone and the airway space were analyzed over various follow-up periods, using cephalometric radiography taken preoperatively, immediately after surgery, eight weeks after surgery, six months after surgery, and one year after surgery. To identify risk factors, multiple regression analysis of age, gender, body mass index (BMI), posterior mandibular movement, and the presence of genioplasty was performed. Results: Inferor and posterior movement of the hyoid bone was observed postoperatively, but subsequent observations showed regression towards the anterosuperior aspect. The airway space also significantly decreased after surgery $(P<0.05)$, and increased slightly up until six months after surgery. The airway space significantly decreased $(\beta=0.47, P<0.01)$ as the amount of mandibular setback increased. However, age, sex, BMI, and presence of genioplasty were not associated with airway reduction.
\end{abstract}

Conclusion: The amount of mandibular set back was significantly associated with postoperative reduction of airway space. It is necessary to establish a treatment plan considering this factor.

Key words: Airway space, Prognathism, Orthognathic surgery, Saggital split ramus osteotomy

\section{Introduction}

Skeletal class III facial patterns can be the result of mandibular prognathism, maxillary deficiency, or a combination of both[1]. Since the soft palate, tongue and hyoid bone, and the associated tissues are attached directly or indirectly to the maxilla and mandible, these tissues are directly affected by movement of the jaw, causing alterations in the pharyngeal area.

Changing the position of the jaw in orthognathic surgery

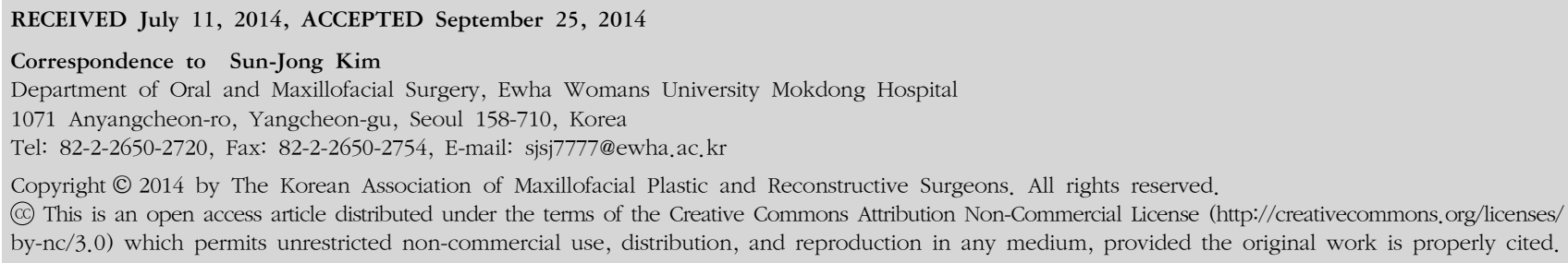


(OS), especially the mandible, the position of the tongue, and the pharyngeal airway space, also causes a change in morphology. The narrowing or widening of the pharyngeal airway space is related to mandibular setback and advancement surgery[2-4].

Lateral cephalometric radiography remains an important imaging tool in surgical planning. It allows the maxillofacial surgeon to plan the surgery and collect relevant information about the hard and soft tissue structures, as well as the airway space. Although radiographic imaging provides only two-dimensional images for the evaluation of the pharyngeal airway, it is still used to evaluate sleep disorders and skeletal deformities[2,3,5].

The advantages of cephalometric radiography include its wide availability, simplicity, low expense, and ease of comparison with studies that also used this method[5,6]. There are significant differences in cephalometric measurements between patients with obstructive sleep apnea syndrome (OSAS) and those without[6]. Many studies based on lateral cephalometric analysis find that the most frequent anatomic alterations associated with OSAS are posterior positioning of the hyoid bone and the base of the tongue, resulting in pharyngeal narrowing. These studies focused on the effect of OS on the pharyngeal airway space[2,3,7].

Orthognathic advancement surgery is an effective option for the treatment of OSAS[8], because it enlarges the pharyngeal airway space and tightens the upper airway muscles and tendons by advancing the osseous origins. Conversely, the pharyngeal airway space sometimes narrows after mandibular setback, with a risk of developing OSAS. This risk has been confirmed in patients who underwent OS for mandibular setback, raising awareness of research of airway narrowing[2,3,7].

The benefits produced by OS have been well described in patients with congenital or acquired sleep disorders, but the effects in patients without this condition is problematic, and pertinent research is sparse[2,9,10]. According to Foltán et al.[10], some studies have found that the alterations to be temporary, whereas others consider them permanent and prone to future worsening, owing to mandibular setback surgery with or without association with maxillary advancement. A long-term observational study to analyze the changes in the airway space is suggested for surgeries involving mandibular setback[7]. In addition, changes in the position of the hyoid bone after OS are widely documented, but whether it returns to the original position during the postoperative follow-up period is still under debate[11-13]. More study is needed to find what can affect the changes of the airway space and the position of the hyoid bone. In light of this, the purposes of this study were as follows: 1) To evaluate the changes of the airway space and the position of hyoid bone after OS (mandibular setback surgery) in patients who had skeletal class III facial pattern without any kind of sleep disorder; 2) To identify possible risks for these changes.

\section{Materials and Methods}

\section{Study subjects}

To address the research hypothesis, the authors conducted a retrospective study evaluating the measurements on lateral cephalometric radiographs of subjects with a Class III facial pattern.

Patients who underwent OS with backward movement of the mandible through the bilateral sagittal split ramus osteotomy (BSSRO) technique in the Department of Oral and Maxillofacial Surgery at Ewha Womans University Medical Center during the period from January 2003 to July 2011 were included in this study. The same surgeon operated on every case.

We considered only mandibular movement and excluded the maxilla and mandible combined double jaw surgery. The advance movement of chin was included and backward movement excluded in the genioplasty. Surgeries were performed under general anesthesia with rigid internal fixation using titanium plates and screws.

The patients ranged in age from 19 to 35 years old, were healthy, no known medical history, no signs of habitual sleep disorder, and no history of previous OS. Patients who did not have a postoperative follow up period of more than one year were excluded. The Institutional Review Board of the Ewha Medical Center, Seoul, Korea reviewed and approved this study and access to patients' records. 


\section{Methods}

1) Lateral cephalometric radiography and measurement

Lateral cephalometric radiographs were taken using the Ortho Stage AUTO III N CMR (Asahi Co., Seto City, Japan). The head was fixed with a cephalostat in the natural head position with maximum intercuspitation conditions. A tube voltage of $73 \mathrm{kVp}$ and a current of $15 \mathrm{~mA}$ were set as the recording conditions, and the recording time was 7.4 seconds. Lateral cephalometric radiographs were taken before surgery (T0), immediately after surgery (T1), two months after surgery (T2), six months after surgery (T3), and one year after surgery (T4). The cephalometric radiographs were converted to digital image communication in medicine (DICOM) files, reconstructed using the V-ceph program (Ver. 7.0; Osstem, Seoul, Korea) for calibration, and analyzed by an orthodontist.

\section{2) Measurement points and measurements}

(1) Baseline (reference plane): The horizontal reference line (horizontal reference plane, HRP) lies above the sella to nasion $(\mathrm{SN})$ plane over 7 degrees. The vertical reference line is perpendicular to the HRP, and passes through the sella point (vertical reference plane, VRP).

To calibrate the amount of mandibular set back movement, the distance from the VRP to point $\mathrm{B}$ was measured (Fig. 1).

(2) Hyoid bone: Measurements of the distance of the hyoid bone from different landmarks include the following: HRP-Hy, measurement of the horizontal distance from the most anterior-superior position of the hyoid bone to the HRP; VRP-Hy, measurement of the horizontal distance from the most anterior-superior position of the hyoid bone to the VRP; C-Hy, measurement of the distance from the most anterior-superior position of the hyoid bone to the most posterior point of the mandibular symphysis.

(3) Airway space: Airway space was measured as the horizontal distance from the most superior point of the second cervical spine to the posterior of tongue.

\section{Assessment methods and statistical analysis}

1) Changes in the airway space and the hyoid bone after surgery

Measurements were performed T0, T1, T2, T3, and T4.
The degree of change in the hyoid bone and the airway space was evaluated at each exam. Null hypotheses of no change were tested using a linear mixed model for verification, with repeated measures.

\section{2) Evaluation of risk factors}

To evaluate the risk factors associated with the changes in the airway space after OS, multiple linear regression analysis was performed of the patient's age, sex, amount of mandibular setback, body mass index (BMI), and presence of genioplasty. Statistical analysis was performed using IBM SPSS Statistics 20 Software (IBM Co., Armonk, NY, USA).

\section{Results}

The lateral cephalometric radiographs of 50 patients (21 males and 29 females) with a mean age of 24.1 years (range

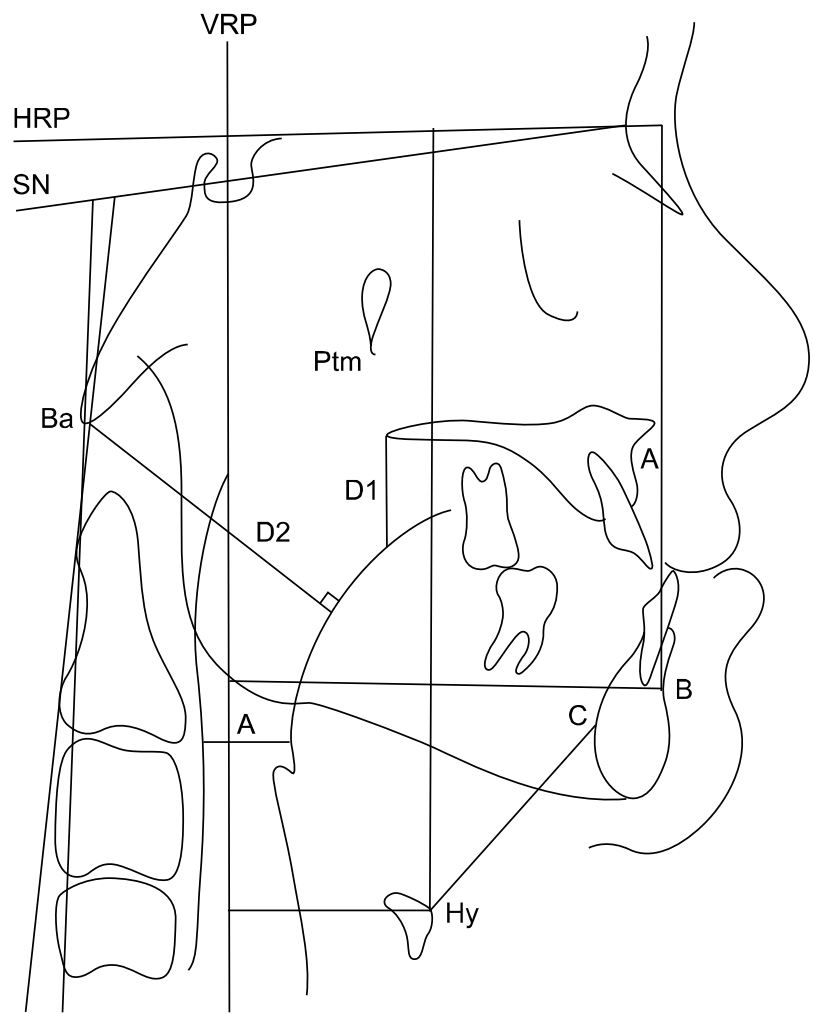

Fig. 1. Craniocervical and hyoidal measurement and pharyngeal airway space measurement. VRP, vertical reference plane; HRP, horizontal reference plane; SN, sella to nasion; Ptm, pterygomaxillary fissure; $\mathrm{Ba}$, basion; $\mathrm{DI}$, distance from posterior nasal spine to tongue; $\mathrm{D} 2$, distance from basion to tongue; $\mathrm{A}$, pharyngeal airway space; B, point B; C, most posterior point of the symphysis of mandible; Hy, hyoid bone position. 
$20 \sim 33$ years) and a mean BMI of 21.65 (range 16.3 31.75) were included in the sample set. The changes observed are included in the sections that follow.

\section{Changes of hyoid bone position at each period of time}

\section{1) Change of vertical position}

Post-operative downward movement of the hyoid bone with reference to the HRP and the hyoid bone (Hy) was observed. The preoperative (T1) had a distance of $117.06 \pm 8.45 \mathrm{~mm}$ (T0), which increased to $120.82 \pm 9.91$ $\mathrm{mm}$ (T1). The increase observed at T1 consistently showed a decreasing trend across patients (Table 1).

\section{2) Change of horizontal position}

The vertical distance of hyoid from the baseline changed from $14.07 \pm 6.70 \mathrm{~mm}$ (T0) to $9.71 \pm 6.26 \mathrm{~mm}$ (T1), and this pattern continued for six months after surgery.

\section{3) Change of the hyoid bone position relative to the} symphysis of the mandible

The distance between the hyoid bone and the most posterior point of the symphysis of the mandible increased from $40.69 \pm 4.68 \mathrm{~mm}$ (T0) to $41.94 \pm 5.56 \mathrm{~mm}$ (T1) as the hyoid bone moved backward postoperatively, but decreased to $39.72 \pm 4.97 \mathrm{~mm}$ (T3) six months post-surgically.

\section{Changes of the airway space}

The preoperative airway space of $18.55 \pm 4.17 \mathrm{~mm}$ (T0) decreased to $14.38 \pm 4.08 \mathrm{~mm}$ post-surgically (T1). Over a six month period, gradual recovery of the airway space was observed, recovering to $16.44 \pm 3.64 \mathrm{~mm} \mathrm{(T3).}$ However, the passage of an additional six months did not lead to more recovery, only reaching a value of $16.58 \pm 3.60$ $\mathrm{mm}(\mathrm{T} 4)$. Full recovery to the original volume was not observed (Table 2).

Table 2. Airway space changes $(n=50)$

\begin{tabular}{ccc}
\hline & Mean \pm standard deviation & Number \\
\hline T0 & $18.55 \pm 4.17$ & 50 \\
T1 & $14.38 \pm 4.08$ & 50 \\
T2 & $15.49 \pm 3.81$ & 50 \\
T3 & $16.44 \pm 3.64$ & 50 \\
T4 & $16.58 \pm 3.60$ & 50 \\
\hline
\end{tabular}

T0, before surgery; T1, immediately after surgery; T2, two months after surgery; T3, six months after surgery; T4, one year after surgery.

Table 1. Change of hyoid bone position $(n=50)$

\begin{tabular}{|c|c|c|c|c|c|c|}
\hline & \multicolumn{2}{|c|}{ HRP-Hy } & \multicolumn{2}{|c|}{ VRP-Hy } & \multicolumn{2}{|c|}{$\mathrm{C}-\mathrm{Hy}$} \\
\hline & Mean & SD & Mean & SD & Mean & SD \\
\hline T0 & $117.06^{\mathrm{a}}$ & 8.45 & $14.07^{\mathrm{a}}$ & 6.70 & 40.69 & 4.68 \\
\hline $\mathrm{T} 1$ & $120.82^{b}$ & 9.91 & $9.71^{\mathrm{b}}$ & 6.26 & 41.94 & 5.56 \\
\hline T2 & $118.46^{\mathrm{a}}$ & 9.35 & $11.42^{\mathrm{a}, \mathrm{b}}$ & 5.99 & 40.67 & 5.48 \\
\hline T3 & $117.36^{\mathrm{a}}$ & 9.45 & $12.32^{\mathrm{a}, \mathrm{b}}$ & 5.95 & $39.72^{a}$ & 4.97 \\
\hline $\mathrm{T} 4$ & $117.42^{a}$ & 9.77 & $12.59^{\mathrm{a}}$ & 5.93 & $39.64^{a}$ & 4.88 \\
\hline
\end{tabular}

HRP-HY, horizontal reference plane to hyoid; VRP-Hy, vertical reference plat to hyoid; C-Hy, most posterior point of the symphysis of mandible to hyoid; T0, before surgery; T1, immediately after surgery; T2, two months after surgery; T3, six months after surgery; $\mathrm{T} 4$, one year after surgery.

${ }^{\mathrm{a}}$ The difference of the estimated average compared to $\mathrm{T} 1$ in each time period was considered statistically significant $(P<0.05)$. ${ }^{\mathrm{b}} \mathrm{The}$ difference of the estimated average compared to T0 in each time period was considered statistically significant $(P<0.05)$.

Table 3. Factors associated with airway change

\begin{tabular}{|c|c|c|c|c|c|c|}
\hline & \multicolumn{3}{|c|}{ Univariate analysis } & \multicolumn{3}{|c|}{ Multivariate analysis ${ }^{a}$} \\
\hline & $\beta$ & SE & $P$-value & $\beta$ & SE & $P$-value \\
\hline Age & 0.09 & 0.05 & ns & 0.07 & 0.05 & ns \\
\hline Gender & -0.27 & 0.59 & ns & -0.09 & 0.56 & ns \\
\hline Body mass index & -0.01 & 0.09 & ns & -0.04 & 0.08 & ns \\
\hline Amount of set-back & $0.46^{\mathrm{b}}$ & 0.11 & $>0.001^{b}$ & $0.47^{\mathrm{b}}$ & 0.52 & $>0.001^{b}$ \\
\hline Genioplasty & 0.24 & 0.58 & ns & -0.29 & 0.54 & ns \\
\hline
\end{tabular}

SE, standard error; ns, none significant.

${ }^{a}$ Mutually adjusted linear regression model. ${ }^{b}$ Statistically significant $(P<0.05)$. 


\section{Risk factors affecting the airway space changes}

Variable potentially affecting airway space changes after OS were evaluated. These were age, gender, amount of setback, BMI and whether or not genioplasty was performed. Multiple regression analysis showed that the amount of set-back has a statistically significant effect on the change in airway spaces, Ts (T1-T0) $(\beta=0.46, P<0.001)$ (Table 3).

\section{Discussion}

Skeletal changes of the jaw due to OS also affect the size of the airway space. Several studies have assessed the changes in the airway space and the position of the hyoid bone, as well as the physiologic reflex mechanism for airway maintenance after skeletal movement in subjects with a skeletal class III facial pattern who underwent OS[11,13,14].

Eggensperger et al.[15] evaluated the lateral radiographs of 32 patients who underwent mandibular setback surgery, taken at one week, six months, and 12 months postoperatively, observing a continuous decrease in the nasopharynx and oropharynx space. Hochban et al.[16], Saitoh[17], and Tselnik and Pogrel[11] also reported decreases in the pharyngeal airway space after mandibular setback surgeries. Demetriades et al.[6] found similar results, and noted a setback of five $\mathrm{mm}$ as potentially critical for the development of obstructive sleep apnea. Chung and Lee[18], Lee[19] and Kim et al.[20] reported that oropharyngeal space decreased postoperatively, with the decrease maintained in the months post-surgery. Marşan et al.[2] found an increase in the nasopharynx with no alterations of the hypopharynx in patients with a Class III facial pattern who underwent bimaxillary surgery. Hasebe et al.[21] did not find any differences in the pharyngeal airway space between the mandibular setback surgery group and bimaxillary surgery group. Pereira-Filho et al.[5] found an increase in the nasopharyngeal space with long-term maintenance, an increase in the oropharynx with relapse in the long term, and a decrease in the hypopharynx with long term maintenance for bimaxillary surgery in patients with a Class III pattern.

Thus, results are conflicting about the changes of the airway space after OS. These variations may arise from the landmarks used for measuring the airway space, which can be different among studies.

In this study, the airway space changed from the T0 value of $18.55 \pm 4.17 \mathrm{~mm}$ to the $\mathrm{T} 1$ value of $14.38 \pm 4.08$ $\mathrm{mm}$, showing the greatest reduction in airway space. This reduction recovered gradually to $16.58 \pm 3.60$ at $\mathrm{T} 4$, but complete recovery was not observed, and a reduction of about $11 \%$ compared to the preoperative airway space measurement was still present.

After mandibular setback surgery the hyoid bone may move inferiorly[13,22,23], both inferiorly and posteriorly[14,15,24,25], and both inferiorly and forward[21]. Some studies also report that the hyoid bone returns to its original position, maintaining the airway resistance[7,11-13,15,24,25].

These changes can occur because of the physiologic reflex mechanism for maintaining the airway space. In this study, we observed posterior and inferior movement of the hyoid bone after mandibular setback surgery, and found anterior and superior movement of hyoid bone towards the original position one year following surgery. However, like the airway change mentioned above, these movements could not return the hyoid bone to its original position.

To assess the risk factors associated with changes in the airway space after OS, age, gender, amount of mandibular set back, BMI, and genioplasty were statistically analyzed. Only setback was identified as a significant factor Ts (T1-T0). The amount of mandibular setback is a statistically significant ( $\beta=0.46, P<0.001)$ risk factor affecting the airway space changes, both by univariate and by multiple regression analysis $(P<0.001)$.

BMI is usually categorized, but we used it as a continuous variable, because most of the patients were in the normal range, and we wished to assess the linear risk of this factor. We thought that continuous variables were appropriate for reliable results statistically.

A relapse occurred in the airway space postoperatively and the gap between each time period (T2-T0, T3-T0) were decreased. The authors performed the same analysis for the time of T2-T0 and T3-T0 for each risk factor, none of which were statistically significant (data not shown).

Further research on the other risk factors affecting the airway space changes associated with OS are needed especially the direction of mandibular set-back, original airway 3-dimensional dimension, and will benefit from technologic developments. 


\section{Conclusion}

The purpose of this study was to evaluate the changes of the airway space and the position of the hyoid bone occurring after mandible set-back surgery, which can be followed by a regression phenomenon to the original position, and to identify the risk factors associated with decreased airway space. To analyze the changing pattern of the hyoid bone and the airway space, lateral cephalometric radiographs which were taken T0, T1, T2, T3, and T4 were analyzed. At each exam, the position of the hyoid bone and the changes in the airway space were measured, and the following conclusions were drawn.

1. After mandible set-back surgery, the hyoid bone moved downward and backward, with subsequent forward and upward movement towards the original position in the postsurgical observation period, but did not return to the exact original position.

2 . The airway space decreased significantly after surgery $(P<0.05)$ and gradually increased up to six months, after which significant improvement was not observed.

3. Among the risk factors affecting the decrease in the airway space after OS, the amount of mandibular setback was the only significant risk factor $(P<0.05)$. Other factors such as age, sex, BMI, and genioplasty did not differ significantly.

\section{Acknowledgements}

This study was supported by the Ewha Womans University Research Grant of 2014-0643.

\section{References}

1. Obwegeser HL. Surgical correction of small or retrodisplaced maxillae. The "dish-face" deformity. Plast Reconstr Surg 1969; 43:351-65.

2. Marşan G, Vasfi Kuvat S, Oztaş E, Cura N, Süsal Z, Emekli U. Oropharyngeal airway changes following bimaxillary surgery in Class III female adults. J Craniomaxillofac Surg 2009; 37:69-73.

3. Sears CR, Miller AJ, Chang MK, Huang JC, Lee JS. Comparison of pharyngeal airway changes on plain radiography and cone-beam computed tomography after orthognathic surgery. J Oral Maxillofac Surg 2011;69:e385-94.

4. Marşan G, Cura N, Emekli U. Changes in pharyngeal (airway) morphology in Class III Turkish female patients after man- dibular setback surgery. J Craniomaxillofac Surg 2008;36:341-5.

5. Pereira-Filho VA, Castro-Silva LM, de Moraes M, Gabrielli MF, Campos JA, Juergens P. Cephalometric evaluation of pharyngeal airway space changes in class III patients undergoing orthognathic surgery. J Oral Maxillofac Surg 2011;69:e409-15.

6. Demetriades N, Chang DJ, Laskarides C, Papageorge M. Effects of mandibular retropositioning, with or without maxillary advancement, on the oro-naso-pharyngeal airway and development of sleep-related breathing disorders. J Oral Maxillofac Surg 2010;68:2431-6.

7. Kawakami M, Yamamoto K, Fujimoto M, Ohgi K, Inoue M, Kirita $\mathrm{T}$. Changes in tongue and hyoid positions, and posterior airway space following mandibular setback surgery. J Craniomaxillofac Surg 2005;33:107-10.

8. Goodday R. Diagnosis, treatment planning, and surgical correction of obstructive sleep apnea. J Oral Maxillofac Surg 2009;67:2183-96.

9. Greco JM, Frohberg U, Van Sickels JE. Long-term airway space changes after mandibular setback using bilateral sagittal split osteotomy. Int J Oral Maxillofac Surg 1990;19:103-5.

10. Foltán R, Hoffmannová J, Pavlíková G, et al. The influence of orthognathic surgery on ventilation during sleep. Int J Oral Maxillofac Surg 2011;40:146-9.

11. Tselnik M, Pogrel MA. Assessment of the pharyngeal airway space after mandibular setback surgery. J Oral Maxillofac Surg 2000;58:282-5.

12. Enacar A, Aksoy AU, Sençift Y, Haydar B, Aras K. Changes in hypopharyngeal airway space and in tongue and hyoid bone positions following the surgical correction of mandibular prognathism. Int J Adult Orthodon Orthognath Surg 1994;9: 285-90.

13. Achilleos S, Krogstad O, Lyberg T. Surgical mandibular setback and changes in uvuloglossopharyngeal morphology and head posture: a short- and long-term cephalometric study in males. Eur J Orthod 2000;22:383-94.

14. Kawamata A, Fujishita M, Ariji Y, Ariji E. Three-dimensional computed tomographic evaluation of morphologic airway changes after mandibular setback osteotomy for prognathism. Oral Surg Oral Med Oral Pathol Oral Radiol Endod 2000;89: 278-87.

15. Eggensperger N, Smolka W, Iizuka T. Long-term changes of hyoid bone position and pharyngeal airway size following mandibular setback by sagittal split ramus osteotomy. J Craniomaxillofac Surg 2005;33:111-7.

16. Hochban W, Schürmann R, Brandenburg U, Conradt R. Mandibular setback for surgical correction of mandibular hyperplasia--does it provoke sleep-related breathing disorders? Int J Oral Maxillofac Surg 1996;25:333-8.

17. Saitoh K. Long-term changes in pharyngeal airway morphology after mandibular setback surgery. Am J Orthod Dentofacial Orthop 2004;125:556-61.

18. Chung DH, Lee KS. A study on changes of airway, tongue, and hyoid position following orthognathic surgery. Korean J Orthod 1998;28:487-98.

19. Lee SH. A study of relapse and position of hyoid bone following orthognathic surgery. J Korean Acad Maxillofac Plast Reconstr Surg 1991;13:476-90.

20. Kim HY, Choi HG, Kim EK, Kim JG. The effects of mandibular setback osteotomy on the oropharyngeal airway space in man- 
dibular prognathic patients. Korean J Orthod 1997;27:733-41.

21. Hasebe D, Kobayashi T, Hasegawa M, et al. Changes in oropharyngeal airway and respiratory function during sleep after orthognathic surgery in patients with mandibular prognathism. Int J Oral Maxillofac Surg 2011;40:584-92.

22. Takagi Y, Gamble JW, Proffit WR, Christiansen RL. Postural change of the hyoid bone following osteotomy of the mandible. Oral Surg Oral Med Oral Pathol 1967;23:688-92.

23. Samman N, Tang SS, Xia J. Cephalometric study of the upper airway in surgically corrected class III skeletal deformity. Int J Adult Orthodon Orthognath Surg 2002;17:180-90.

24. Wickwire NA, White RP Jr, Proffit WR. The effect of mandibular osteotomy on tongue position. J Oral Surg 1972;30:184-90.

25. Lew KK. Changes in tongue and hyoid bone positions following anterior mandibular subapical osteotomy in patients with Class III malocclusion. Int J Adult Orthodon Orthognath Surg 1993;8:123-8. 